\title{
EL ENCABALGAMIENTO EN LA POESÍA DE FRAY LUIS DE LEÓN
}

Las doctrinas poéticas de los tratadistas españoles del Renacimiento son, en verdad, escasas y tardías ${ }^{1}$. Antes de 1580 , y con la sola excepción ilustre del Brocense, apenas hay nada que pueda compararse a ciertas observaciones sobre la lengua literaria desperdigadas en las Prose della volgar lingua (1525), de Bembo, ni al tratado Del modo di comporre in versi nella lingua italiana que Gerolamo Ruscelli colocó en 1559 al frente de su Rimario della lingua italiana, ni a los Poetices libri septem (1561), de Scaligero, o a la Poetica toscana (1563), de Minturno 2. Tampoco hubo, antes de Herrera, creadores que hicieran del acto literario motivo de reflexión teórica. Ni el Prohemio de Santillana ni los tratados de Villena, Juan del Encina o Argote de Molina pueden situarse en el mismo plano que el Abregé de l'art poétique françoise (1565), de Ronsard, o las afirmaciones de este mismo poeta en el prólogo a su Franciade (1572); y mayor distancia todavía los separa de la obra térica

1 Además de la clásica Historia de las ideas estéticas de Mentendez Pelayo, pueden consultarse las muy provechosas páginas de A. Vilanova, «Preceptistas de los siglos XVI y XVII», en Historia general de las literaturas hispanicas, III, págs. 567. 694; también J. Rico VeRDU, La retórica española de los siglos XVI y XVII, Madrid, CSIC, 1973.

2 Por no citar los trabajos anteriores de humanistas como Pontano o Salutari sobre la poesía latina, con muy agudos análisis de las estructuras métricas. Cfr. G. Martellotrt, «Critica metrica del Salutari e del Pontano», en Critica e storia letteraria, Studi offerti a Mario Fubini, Padova, Liviana Editrice, 1970, I, págs. 352 . 373. 
de Tasso, especialmente de sus discursos sobre el poema heroico o su comentario acerca de un soneto de Giovanni della Casa. Para encontrar una summa teórica que sea, a la vez, un amplio repertorio de artificios literarios, habrá que aguardar a las Anotaciones de Herrera (1580), piedra fundamental de la poética española ${ }^{3}$. Allí habla el poeta sevillano, siguiendo de cerca a Ruscelli, del efecto producido por el «rompimiento» del verso, fenómeno para el cual había acuñado ya Ronsard el término enjambement. A juicio de Herrera, el hecho de que el final del verso no coincida con el final del enunciado

no es vicio, sino virtud, y uno de los caminos principales para alcanzar la alteza y hermosura del estilo; como en el heroico latino, que romper el verso es grandeza del modo de decir. Refiero esto porque se persuaden algunos que nunca dicen mejor que cuando siempre acaban la sentencia con la rima; e oso afirmar que ninguna mayor falta se puede casi hallar en el soneto que terminar los versos de este modo, porque aunque sean compuestos de letras sonantes y de sílabas llenas casi todas, parecen de muy humilde estilo y simplicidad, no por flaqueza y desmayo de letras, sino por sola esta igual manera de paso, no apartando algún verso; que yendo todo entero a acabarse en su fin, no puede tener alguna cumplida gravedad, ni alteza, ni hermosura de estilo, si bien concurriesen todas las otras partes. Pero cuando quiere alguno acompañar el estilo conforme con la celsitud y belleza del pensamiento, procura desatar los versos, y muestra con este deslazamiento y partición cuánta grandeza tiene $\mathbf{y}$ hermosura en el sujeto, en las voces $\mathbf{y}$ en el estilo, porque lo hace levantado, compuesto y bellísimo en la forma y figura del decir esta división, y lo aparta de la vulgaridad de los otros; mas este rompimiento no ha de ser continuo, porque engendra fastidio la perpetua semejanza 4 .

Naturalmente, Fray Luis de León no necesitó aguardar a las Anotaciones de Herrera para apreciar en todo su valor posible el uso del encabalgamiento. Pudo hacerlo antes en los tratadistas italianos, y más todavia en el uso de poetas como Della Casa, Bembo o Garcilaso. No debieron de serle desconocidas tampoco las teorías métricas latinas acerca del colon y el comma ${ }^{5}$, base lejana de la esticomitia y el enca-

3 Cfr. J. Almeida, La critica literaria de Fernando de Herrera, Madrid, Gredos, 1976.

4 Cito por la ed. de A. Gallego Morell, Garcilaso de la Vega y sus comentaristas (Granada, Universidad, 1966), con algún pequeño retoque en la puntuación. La cita se halla en la pág. 284.

5 Cfr. A. Fortunatiano, Ars, 6 (ed. KeIL, Gram. lat., VI, pág. 282), o Mario VicTORINo, De metris Horat., id., pág. 184; SERGIo, Expl. in Donatum (ed. Keil, IV, pág. 485); y la nítida caracterización de BEDA, De arte metrica: a Item ubi post duos pedes superest syllaba, comma dicitur; ubi post duos pedes nihil remanet, colon vocatur» (ed. KBIL, Gram. lat., VII, pág. 246, 1. 18). 
balgamiento en la métrica romance. Y si tanteó el efecto de ciertas fórmulas encabalgadas de insólita audacia, como miserable/mente o vana/mente, arriesgándose a la reprobación de los preceptistas ${ }^{6}$, fue porque halló modelos de suficiente autoridad en poetas latinos ${ }^{7}$. Por otra parte, las observaciones de Ruscelli, Herrera o Tasso acerca del encabalgamiento son sumamente genéricas. Se invoca la «altezza e leggiadriaw del estilo, como hace Ruscelli y adopta Herrera, o se dice que los versos encabalgados "fanno il parlar magnifico e sublime", como asevera Tasso, aunque luego sus encabalgamientos «non adempiono sempre e soltanto la funzione di cui egli parla», según Fubini ${ }^{8}$. $Y$ con razón, porque la función y el valor de los encabalgamientos son siempre contextuales, y sólo una peligrosa simplificación podría atribuirles un significado único y permanente. No contó Fray Luis con una casuística previa a la que amoldar sus experiencias. Hubo de valerse, como cualquier poeta innovador y enemigo de recetas, de su aguda conciencia linguística para calibrar el efecto de cada «rompimiento* antes de decidirse a vulnerar la rítmica apacibilidad del ajuste entre sintaxis y metro. Y la práctica del encabalgamiento fue marcando así, poco a poco, ciertas líneas directrices, tendencias de uso que el análisis debería esclarecer y que forman ya parte de la peculiar poética de Fray Luis, para quien, sin duda, el encabalgamiento no constituyó nunca un mero artificio elegante, sino un instrumento expresivo más del verso. En las páginas que siguen trataremos de esbozar, a manera de sugerencias para un desarrollo más amplio, algunas de esas líneas maestras sobre las cuales erigió Fray Luis su portentosa creación.

Hay en primer lugar un conjunto de casos, con encabalgamientos de diferente magnitud, en que la partición del enunciado en más de un verso ayuda a subrayar una distancia, tanto espacial como temporal. Al agruparse en dos unidades métricas distintas, los elementos de una y otra quedan situados en planos diversos, y esta forzosa disposición

6 Cfr. A. Quilis, «Los encabalgamientos léxicos en -mente en Fray Luis de León y sus comentaristas*, en Hispanic Review, XXXI, 1963, págs. 22-39, con material incorporado luego al libro Estructura del encabalgamiento en la métrica española, Madrid, CSIC, 1964.

7 A los ejemplos de Horacio aducidos por los comentaristas y recogidos por Quilis en su art. cit., añádanse Catulo, 11, 11: «Gallicum Rhenum horribile aequor ulti / mosque Britannos»; id., 61, 86 ss.: «Flere desine, non tibi, Au/runculeia, periculumst"; Horacio, Sat., I, 2, 62: "quid inter / est in matrona, ancilla peccesne togata»; íd., Sat., I, 9, 51: «...est locus uni / cuique suus». Ovidio desaconseja estos artificios: "Nam pudet in geminos ita nomen findere versus, / desinet ut prior hoc incipiatque minor (Pont., IV, 12, 7).

${ }_{8}$ Studi sulla letteratura del Rinascimento2, Firenze, La Nuova Italia, 1971, página 231. 
gráfica permite recalcar una separación que podía haber quedado enmascarada por la articulación sintáctica. Así, en la oda «De la Magdalena» 9:

¿Qué tienes del pasado tiempo sino dolor? ¿Cuál es el fruto que tu labor te ha dado...?

El encabalgamiento de pasado, la pausa métrica y el espacio en blanco aíslan y distancian la noción al dilatarla imaginativamente. El segundo verso, en cambio, agrupa las nociones 'tiempo', 'dolor' y 'fruto', que caracterizan el presente y aparecen como lejana consecuencia («fruto») de aquel pasado. Un efecto similar, producido con análogos recursos, se había producido ya en el comienzo de la misma oda:

Elisa, ya el preciado

cabello, que del oro escarnio hacía,

la nieve ha variado 10.

Lo esencial en estos versos es la idea del cambio, de la transformación que la edad ha obrado en el sujeto, simbolizada en el emblanquecimiento del cabello, en otro tiempo dorado. El cabello fue, por consiguiente, «preciado» antes, en una época ya lejana. El encabalgamiento refuerza, una vez más, la separación, la distancia temporal entre la juventud y la vejez. Dicho de otro modo: el cabello actual del segundo verso es heredero del preciado de la mocedad, que por eso se menciona en el verso anterior. El efecto estilístico de la anteposición del adjetivo se intensifica al situarse el término en posición final de verso, pero, al mismo tiempo, la distribución de los componentes del sintagma se beneficia del encabalgamiento y del espacio en blanco e introduce entre ambos versos todo el proceso de una juventud perdida.

Puede ocurrir que, además del encabalgamiento, otros artificios aumenten la separación entre los miembros del sintagma, como en este caso:

iOh ya seguro puerto

de mi tan luengo error! $\mathfrak{\text { Oh }}$ deseado

para reparo cierto

del grave mal pasado

reposo alegre, dulce, descansado!

9 Sigo, en general, la ed. del P. Vega, aunque en alguna ocasión me aparte de ella.

10 Así en algunos códices y en la ed. de O. Macrí. El P. Vega ofrece la variante «ha demudado». 
Fray Luis no se ha limitado a encabalgar deseado/reposo, sino que ha introducido una amplia expansión que abarca dos versos. El resultado es que la noción semántica contenida en el atributo deseado se amplía imaginativamente para expresar un deseo mantenido durante mucho tiempo. De nuevo, una considerable distancia cronológica -aqui, la que separa un deseo largamente acariciado y el logro real- se refuerza mediante el apoyo del encabalgamiento. Un caso análogo, aunque de más simple artificio, lo constituye la ruptura «descubre el deseado/ rostro», en la composición "Virgen que el sol más pura» (vv. 18-19); la conjunción de "deseado» y el encabalgamiento equivale a 'largamente deseado'.

La separación de los componentes del enunciado en versos distintos puede marcar también una distancia puramente física, espacial -poco importa que sea real o metafórica-, entre los elementos divididos por la pausa métrica. En la oda a Grial, una serie polisindética de enunciados desemboca, tras el encabalgamiento, en la mención de la «cumbre» distante y elevada a la que se aspira:

Alarga el bien guiado

paso, y la cuesta vence, y solo gana la cumbre del collado...

Si el olvido puede ser también interpretado como una separación entre los hechos anteriores y el presente, sólo salvable merced a la memoria, no puede extrañar que en ciertas organizaciones semánticas gobernadas por el sema 'olvido' se agrupen sus componentes teniendo en cuenta el posible apoyo significativo del encabalgamiento. En la oda a Salinas, el alma, estimulada por la música,

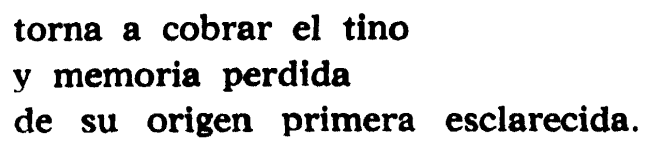

El sintagma «memoria de su origen» posee tal unidad que, en buena lógica, hubiera debido integrarse en un solo verso. La ruptura se debe a la inserción del atributo perdida, que convierte la memoria en 'olvido', y el nuevo significado se refuerza mediante la separación real del olvido y lo olvidado en dos versos distintos. El mismo procedimiento se advierte en la oda "Cuando contemplo el cielo»:

¿Qué mortal desatino

de la verdad aleja asi el sentido

que de tu bien divino

olvidado, perdido,

sigue la vana sombra, el bien fingido? 
Nos encontramos en un contexto muy similar al del ejemplo anterior ${ }^{11}$. El «olvido» del «bien divino» obliga a colocar ambas nociones en dos versos sucesivos. La aparición inmediata de perdido, que inicia un nuevo encabalgamiento, deja los dos adjetivos en el mismo verso, es decir, los iguala, sugiriendo así que quien está olvidado de la divinidad está perdido. Sólo después de la pausa métrica es preciso someterse a los imperativos de la sintaxis, pero resulta ya imposible anular por completo el significado desprendido de la métrica, que se une así al que proporcionan las palabras y su articulación sintáctica.

Razones semánticas son también las que apoyan el encabalgamiento que puede leerse en una de las odas a Felipe Ruiz («En vano el mar fatiga»):

¿Qué vale el no tocado

tesoro, si corrompe el dulce sueño...?

El carácter ajeno y distante del "tesoro» que no llega a ser alcanzado se destaca así, merced a la separación gráfica y métrica, con absoluta nitidez. La «ajena/presencia» ante la que acude Magdalena en la oda «Elisa, ya el preciado» (vv. 53-54), así como la «ajena/casa» de "¿Qué santo o qué gloriosa...?» (vv. 49-50), se hallan en un plano semejante. Un buen número de encabalgamientos luisianos tiene como función esencial la de marcar una distancia, real o subjetiva, cronológica o espacial, entre dos nociones, sin descartar en ciertos casos otros efectos secundarios. Recuérdese el caso de la oda "Alma región lucientew:

¡Oh son! ¡Oh voz! ¡Siquiera

pequeña parte alguna decendiese

en mi sentido, y fuera

de sí el alma pusiese,

y toda en ti, oh Amor, la convirtiese!

La ruptura «fuera / de sí» permite ofrecer la imagen gráfica de una separación ya expresada merced a los valores semánticos del enunciado.

Ahora bien: puesto que el final de verso obliga siempre a una suspensión, por leve que sea, en la fluencia del enunciado ${ }^{12}$, el encabal-

11 Lo que afecta incluso a las rimas y los vocablos seleccionados. En la oda a Salinas, tino rima con (son) divino; aqui, la rima correspondiente a desatino es la del sintagma bien divino. El adjetivo perdida reaparece ahora en perdido. Las analogías contextuales provocan desarrollos léxicos afines.

12 Ya recalcaba Juan del Encina en 1496 que entre verso y verso la lectura se ha de «parar un poquito (apud Mrnéndez Pelayo, Historia de las ideas estéticas en España, Madrid, CSIC, 1962, I, pág. 524. 
gamiento puede estar organizado exclusivamente para detener, destacándolo y alargándolo, el término extremo, que en circunstancias normales resultaría casi borrado por el mayor peso específico de lo que ahora queda como rejet. De ahí que sea frecuente encabalgar adjetivo/ sustantivo, con lo que el miembro inicial recupera una primacía que la sintaxis le había arrebatado. Este alargamiento tiene especial justificación cuando el contenido semántico del término es compatible con la idea de prolongación que implícitamente contiene el espacio en blanco, como en la oda a Salinas:

...y oye alli otro modo

de no perecedera

música, que es de todas la primera.

La perennidad de la música procedente de la «más alta esfera» tiene su equivalencia gráfica e imaginativa en el corte del verso y su correspondiente pausa. De este modo, además, la cualidad prevalece sobre la sustancia; lo esencial no es que el alma oiga una música, sino que ésta sea «no perecedera». Las "inmortales/columnas» de la oda "¿Cuándo será que pueda...?» tienen un fundamento análogo ${ }^{13}$.

La detención del enunciado permite igualmente suscitar un significado equívoco e intencionado que, aun deshaciéndose al proseguir la lectura en el verso siguiente, deja flotando la huella de su primitiva interpretación. Ocurre, sobre todo, en contextos deliberadamente ambiguos, cuyo posible sentido oculto no ha dejado de ser objeto de atención por parte de la crítica. Así acontece con la oda " ¡Oh ya seguro puerto...! $»$, en la que se ha querido ver una reflexión posterior al proceso y encarcelamiento del autor ${ }^{14}$, con alusiones transparentes al comportamiento de los detractores y enemigos del poeta. En los vv. 6 y siguientes de la composición escribe Fray Luis:

Techo pajizo, adonde jamás hizo morada el enemigo cuidado...

13 Lo que no logró percibir, a pesar de su amplitud de criterio, Francisco Llorente, que en su Tratado de la Prosodia española (1846) concluía, tras aducir el ejemplo de Fray Luis: «El poeta debe encerrar un pensamiento en cada verso siempre que pueda, o terminarle en una palabra que represente una idea* (cit. por A. Quilis, Estructura del encabalgamiento..., pág. 23).

14 Cfr. A. Costze, en Revue Hispanique, XLVI, 1919, pág. 244; D. Alonso, «Vida y poesía en Fray Luis de León», en Obras completas, II, Madrid, Gredos, 1973, págs. 829 ss.; O. MAcrt, La poesía de Fray Luis de León, Salamanca, Anaya, 1970, pág. 357; también el P. Vega, en su edic. crítica (Madrid, SAETA, 1955), pág. 512.

LXII. -4 
Aunque el rejet descubre que el término enemigo es un atributo de cuidado, lo cierto es que la interrupción del verso ha sugerido al lector que la palabra es aquí un sustantivo - de carácter colectivo, además - que se refiere abiertamente a los adversarios que promovieron el proceso y la condena del poeta. Fray Luis aprovecha de nuevo esta doble posibilidad del término en un contexto muy similar, perteneciente a la oda « ¿Qué santo o qué gloriosa...?», precisamente en la parte (vv. 71 y ss.) que ya se le antojaba a Llobera "añadida y compuesta, a lo que parece, en la cárcel» ${ }^{15}$ :

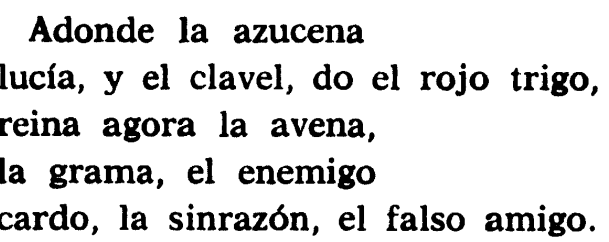

La impresión es aquí semejante a la del caso anterior, y, por añadidura, se refuerza porque la rima apunta una correlación entre el enemigo y el falso amigo del verso inmediato, e insinúa, por tanto, su equivalencia sintáctica. Al «parar un poquito» en la lectura, como aconsejaba Juan del Encina, la construcción "reina [...] el enemigo» ofrece un sentido inmediato que difícilmente puede ser borrado por el contorno metafórico que la envuelve. Una vez más, la posición encabalgada de un elemento léxico proporciona al verso el aditamento de un significado secundario. Como es obvio, las informaciones aportadas por el texto no provienen únicamente del contenido semántico de las palabras.

El encabalgamiento desempeña también una función significativa en los versos 46-47 de la oda « ¡Oh ya seguro puerto...!», cuando el poeta, reelaborando un pasaje de San Juan Crisóstomo ${ }^{16}$, evoca a los «navegantes» del «mar» de la vida humana sometidos a poderosas tempestades:

El otro en la encubierta peña rompe la nave...

Los significados léxicos han determinado aquí decisivamente la organización del enunciado. La roca contra la cual se estrella el metafórico navío estaba oculta a la vista del piloto («encubierta») ${ }^{17}$. Sólo después,

15 En su edic. de Madrid, 1932, I, pág. 528.

16 Cfr. mis Tres estudios sobre Fray Luis de Leon, Salamanca, Universidad, 1978, pág. 43.

17 Un encabalgamiento de otra naturaleza gramatical, pero que guarda cierta analogía con éste, por depender también del sema 'ocultamiento', aparece en la 
provocado ya el naufragio, es posible advertir la situación y la naturaleza del funesto escollo $y$, en consecuencia, identificarlo como «peña* en el verso siguiente. En el fondo, muchos de los valores del blanco y de la pausa métrica son siempre reductibles a espacio y a tiempo; a la noción de distancia, en suma, en cualquiera de sus variedades posibles. He aquí otro caso, de la composición «Virgen que el sol más pura* (vv. 81-83):

Mil olas a porfía

hunden en el abismo un desarmado

leño de vela y remo...

La mención de la nave -leño, según el uso italiano, que, a su vez, constituía un calco semántico del pinus latino ${ }^{18}$ - aparece agrupada en el mismo verso con sus elementos característicos: vela y remo. En cambio, la noción 'hundir' se halla en el verso anterior, en compañía del atributo desarmado. La explicación es simple: la idea de la nave como tal («leño de vela y remo») es independiente de su representación cuando, ya desarbolada, se hunde en el metafórico mar. Lo que «desarmado» se sumerge en las aguas no es, en rigor, un «leño de vela y remo», sino sus restos, y los dos momentos del proceso, con sus rasgos léxicos característicos, se sitúan en versos diferentes.

Sin abandonar la serie de rupturas producidas en el interior del sintagma nominal, recordemos ahora uno de los pasajes más conocidos y citados de la poesía de Fray Luis:

¿Qué descansada vida

la del que huye el mundanal ruido

y sigue la escondida

senda por donde han ido

los pocos sabios que en el mundo han sido!

La «escondida/senda* ha suscitado numerosas interpretaciones en cuanto a su significado ${ }^{19}$, e incluso un análisis del encabalgamiento ${ }^{20}$ : «Son pocos los que se alejan del mundo porque el camino es más estrecho, más difícil de encontrar también; y Fray Luis, para poner de relieve esta dificultad, emplea en el momento preciso el encabalga-

oda «Las Serenas», reelaborando un verso de Virgilio: «Retira el pie, que asconde / sierpre mortal el pradox.

18 Cfr. R. LAPESA, Poetas y prosistas de ayer y de hoy, Madrid, Gredos, 1977, pág. 116.

19 Cfr. «La 'escondida senda' de Fray Luis», en mis Tres estudios sobre Fray Luis de León, cit., págs. 7-36.

20 A. Qun.rs, Estructura del encabalgamiento..., pág. 96. 
miento, utilizando para ello el sirrema nominal cuyo núcleo es la senda por donde van los pocos sabios que han existidox. Parece, no obstante, que la complejidad del encabalgamiento es mayor en este caso, a tono con la densidad especial de la oda en que se encuentra. En primer lugar, «la escondida/senda* se amolda a la misma pauta que «la encubierta / peña ya comentada: el encabalgamiento transcribe el tiempo de búsqueda de la "senda" hasta dar con ella e identificarla. Pero, por otra parte, la rima establece así una conexión entre (senda) escondida y descansada vida, y refuerza la idea de que la una conduce sin interrupción a la otra. Por último, la misma rima -y la pausa métrica, claro está- induce momentáneamente a interpretar «y sigue la escondida (vida)», que sería, en efecto, la de quienes huyen ael mundanal ruido», esto es, la «vida» recatada y honda de la teología mística frente a la más externa de la teología especulativa.

Los encabalgamientos adjetivo/sustantivo, frecuentísimos en la poesía de Fray Luis, sirven al menos -y así ocurre en muchas ocasionespara recalcar lo que la simple inversión en la distribución de los elementos del sintagma sugiere por sí sola: que lo «sustantivo» es justamente lo que va en primer lugar, al margen de la categoría gramatical de la palabra. No tiene otro sentido el hecho, fácilmente comprobable por cualquier lector, de que las rupturas del sintagma nominal se produzcan casi siempre con la anteposición del adjetivo ${ }^{21}$, frente a las escasísimas con adjetivo pospuesto ${ }^{2}$, uso éste normal, en cambio, fuera del encabalgamiento ${ }^{23}$. La posición anterior del adjetivo y la pausa métrica son dos factores suficientes para invertir la habitual jerarquía entre sustantivo y adjetivo y modificar la función ancilar de éste. Cuando, en la oda " $Q$ Qué descansada vida...!», el sujeto lírico expone sus modestas aspiraciones, el adjetivo encabalgado adquiere un inusitado relieve:

21 Anótense, sin ánimo de agotar el repertorio y sin salir de las odas, ejemplos como «pobrecilla/mesa», «ligero/pie», «dura/muerte», «rara/esperanza», «querida/ prenda», "malvado/amor», «fiada/gente», «mortales/fraguas», «lisonjera/vida», «dorado/vaso», «postrero/asensio», «engañosa/Circe», «reñida/guerra», «ameno/verdor», "perdido/error», «nuevo/estilo», «ñudosa/carrasca», «insano/puñal», «generoso/pimpollo», «divinos/gozos», «luciente/verdad», «claro/día», «puro/aire», «gloriosa/virtud», "lucida/noche», «ligero/medio», «maldita/mano», «bendita/alma», «soberano/Hijo», "malvada/fuerza*, *doliente/ánimo».

22 «Ceño/vanamente severo», "torbellino/traidor», "proceso / vario», «ciento / otras».

${ }_{23}$ Formas sin encabalgar, como «lengua lisonjera», «verdad sincera», «ansias vivas», «mar tempestuoso», «día puro», «cantar sabroso», «cuidados graves», "fruto cierto», «cumbre airosa», «fontana pura», «sed insaciable», «son dulce» aparecen ya en la oda " ¿Qué descansada vida...!». 


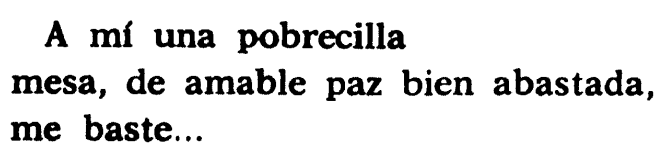

Aun a riesgo de repetir observaciones anteriores, importa destacar que lo esencial de la «mesa» es que sea «pobrecilla», y no en vano el poeta ha utilizado el sufijo con indudable intención afectiva. La exhortación «No te engañe el dorado/vaso» con que se inicia la oda «Las Serenas" constituye un encabalgamiento de más palmario designio aún, si cabe, ya que el peligro del engaño no reside en el «vaso», sino en su llamativa riqueza. En la misma oda, "la engañosa/Circe» pone por delante el carácter de la hechicera, factor relevante en el contexto admonitorio de la composición. Y, en la oda a Grial, el «nuevo/estilo» capaz de igualar al antiguo proclama así, desde su posición destacada, la confianza en su triunfo.

Un examen pormenorizado de estos y otros tipos de encabalgamiento que ofrece la poesía de Fray Luis, imposible de realizar en el breve bosquejo de unas pocas páginas, ilustraría acerca de las pautas constructivas de uno de los poetas más densos y reflexivos del Renacimiento español, a medio camino de una trayectoria de artificios retóricos que se inicia con Garcilaso para concluir en los grandes poetas del Barroco. No hemos calibrado aún adecuadamente las aportaciones de Fray Luis a la lengua literaria del siglo XvI, a pesar de los muy valiosos estudios que han ido abriendo el camino en diversas direcciones. Faltan muchos secretos por descubrir en una producción que sólo en apariencia está exenta de misterios. Unicamente un asedio tenaz y continuo, apoyado en múltiples interrogatorios directos de la obra, puede conducir a nuevos hallazgos de hechos refractarios hasta ahora a cualquier indagación.

Ricardo Senabre 